\title{
Perspectives on our cosmic habitat
}

\author{
Martin J. Rees \\ Institute of Astronomy, University of Cambridge, \\ Madingley Road, Cambridge CB3 0HA, United Kingdom \\ email: mjr36@cam.ac.uk
}

\section{Introduction}

In this talk I shall try to highlight some themes that seem to promise specially interesting progress in the coming decades. I can't claim to be much of a prophet myself, but I'd like to recall someone who was: Arthur C. Clarke. He lived in Sri Lanka, and died in 2007, aged 90. I'd like to discuss three issues that he would have found interesting: space and alien life; galaxies and their origin; and (more speculatively) the size of our universe.

\section{Life in space}

Clarke said his greatest wish was to live long enough to see the discovery of alien life -sadly, he didn't. But he did live long enough to compare some of his predictions with reality -especially regards space. He wasn't of course the first person to think about space travel. Among his precursors was the great Newton. He calculated how fast a cannon-ball would need to be fired if its trajectory were to curve downwards only at the same rate as the Earth's surface curved, so that it stayed in orbit. The required speed vastly surpassed the powers of the artillery of Newton's day. But of course this feat was achieve in 1957 when the Soviet Union launched the first Sputnik.

In the 1960s manned spaceflight went from cornflakes packet to reality. But since then its glamour has faded. Only those who have now reached middle-age can remember the murky TV pictures of Neil Armstrong's 'one small step'. And it's over 35 years since Jack Schmidt and Eugene Cernan, the last men on the Moon, returned to Earth. Many of us then expected a lunar base, even an expedition to Mars, within thirty years. But 2001 didn't resemble Clarke's vision any more than 1984 (fortunately) resembled Orwell's. The race to the Moon was an end in itself -almost just a stunt-driven by superpower rivalry. The impetus was lost. The Apollo program now seems a remote historical episode: young people all over the world learn that America landed men on the Moon, just as they learn that the Egyptians built the pyramids; but the motivations seem almost as bizarre in the one case as in the other.

But of course robotic exploration has burgeoned. Unmanned probes to other planets have beamed back pictures of varied and distinctive worlds. Perhaps the most remarkable triumph of robotics was the Huygens probe, which parachuted onto Saturn's giant moon Titan without any 'real time' control (radio signals from Earth would take hours to reach Titan).

I hope that, within a century, the entire solar system will have been explored and mapped by flotillas of tiny robotic craft. Space will also be pervaded by robots and 'fabricators', allowing large construction projects, using raw materials that need not come from Earth. But will people have followed them?

When asked about the case for sending people into space, my answer is that as a scientist or practical man I'm against it, but as a human being I'm in favour. The 
practical case for manned spaceflight gets ever-weaker with each advance in robotics and miniaturisation. I nonetheless hope that some people now living will walk on Mars. But it will be hazardous: there must be an overt acceptance of this. A rôle model for future astronauts should surely be the great explorers of earlier centuries, willingly taking high risks. Remember that nowhere in our Solar system offers an environment even as clement as the Antarctic or the top of Everest. Space doesn't offer an escape from Earth's problems.

But let us now widen our gaze beyond our Solar System -to other stars. Since the 1990s we've learnt something that's made the night sky far more interesting. Stars aren't mere twinkling 'points of light'. They're orbited by retinues of planets, just like the Sun is. Most are not detected directly but by the way they cause the central star's position to wobble around the barycentre. Already hundreds of extra-solar planets have been found.

The evidence up till now pertains to 'giant' planets -objects the size of Saturn or Jupiter. But we'll be especially interested in possible 'twins' of our Earth -planets the same size as ours, orbiting other Sun-like stars, on orbits with temperatures such that water neither boils nor stays frozen. Detecting Earthlike planets is a real challenge.

We can see their shadow - a star dims slightly when a planet moves in front of itwhen a planet is 'in transit'. The Kepler spacecraft, due for launch in March, could find dozens. But we'd really like to see these planets directly - not just their shadows. And that's hard. To realise just how hard, suppose an alien astronomer with a powerful telescope was viewing the Earth from (say) 30 light years away -the distance of a nearby star. Our planet would seem, in Carl Sagan's phrase, a 'pale blue dot', very close to a star (our Sun) that outshines it by many billions: a firefly next to a searchlight. The shade of blue would be slightly different, depending on whether the Pacific ocean or the Eurasian land mass was facing them. The aliens could infer the length of the 'day', the seasons, the gross topography, and the climate. By analysing the faint light, they could perhaps even infer that it had a biosphere.

Within 20 years, telescope arrays in space, or a new generation of huge telescopes on the ground, will detect planets the same size as our Earth, orbiting other Sun-like stars. Would there be life on any of them? We know too little even to assess whether this is likely or unlikely. We don't understand how life began here on our Earth -which now offers a habitat for an enormous variety of creatures, from slime mould to monkeys (and of course humans as well). Searches for life beyond our Solar System will justifiably focus on Earthlike planets orbiting long-lived solar-type stars. But maybe we should be less parochial. Could there be balloon-like creatures floating in the dense atmospheres of Jupiter-like planets? And science fiction writers have other ideas. (It is better, incidentally, to read first-rate science fiction, like Arthur C. Clarke's, than second-rate science.) The quest for alien life is a fascinating challenge: its outcome will influence our concept of our place in nature as profoundly as Darwinism has over the last 150 years. But we shouldn't hold our breath for success. Even if simple life is common, it is of course a separate question whether it's likely to evolve into anything we might recognize as intelligent or complex. The "SETI@home" programme, involving literally millions of people, is a fine example of the 'citizen science' that Baruch Blumberg discussed in the previous talk. Perhaps we'll one day detect a signal from space that's clearly artificial. Even if it's very boring, -a list of prime numbers of the digits of $\pi$, it would carry the momentous message that concepts of logic and physics (if not consciousness) aren't limited to the hardware in human skulls. On the other hand, perhaps such searches will fail -perhaps there's no galactic community to 'plug in' to. Earth's intricate biosphere may be unique. That may be disappointing. But it would have its upside: it would entitle us to be less cosmically 
modest. Our tiny planet could then be the most important place in the Galaxy -perhaps even a 'seed' from which life could spread through the entire Galaxy.

\section{Stars and galaxies}

Now some general thoughts on our cosmic habitat and how it emerged. Our Solar System formed by condensing from a dusty, slowly spinning cloud. We see places (the Eagle Nebula, for instance) where stars (and probably planets) are still forming now. And we see stars dying, some as planetary nebulae -as our Sun will in 6 billion yearsand some explosively, as supernovae. Our galaxy is a kind of ecosystem where gas is processed and recycled through successive generations of stars. This process generates, from pristine hydrogen, the elements of the periodic table. All the carbon, oxygen and iron on Earth, and in our bodies is ash from long-dead stars. We are the 'nuclear waste' from the fusion power that makes stars shine. We can understand why carbon and oxygen are common; why gold and uranium are rare. The atoms that we are made of came from many different stars, that lived long ago, far away in the Milky Way. The cosmos is part of our environment in a very intimate sense. This is a concept that has wide appeal.

Let us now enlarge our horizons further. As later speakers will discuss, we've made immense progress in understanding galaxies. We know that, in addition to the stars and gas that we see, they contain dark matter, and that black holes lurk at their centres. Largescale surveys not only offer large samples of galaxies for statistical analysis, but allow us to study how galaxies are clustered. And we can probe far back into the past. Amazing images, like the Hubble Ultra-Deep Field, reveal many hundreds of faint smudges even in a small patch of sky less than a hundredth of the area covered by a full Moon. Each faint smudge of light is actually an entire galaxy, which appears so small and faint because of its huge distance. The light from these remote galaxies set out up to 12 billion years ago. They're being viewed when they have only recently formed. Some consist mainly of glowing diffuse gas that hasn't yet condensed into stars. When we look at Andromeda, we might wonder whether there are aliens looking back at us. Perhaps there are. But the light we see from these ultra-remote galaxies set out before there'd been time for stars to forge the silicon, carbon and oxygen needed even to make planets, so there's scant chance of life back then.

The brightest objects for spectroscopic study, and the best current probes of the universe back to redshifts of 6 , are the quasars. Their existence proclaims clearly that a great deal had happened by the time our universe was 0.1 of its present age. Some large galaxies had already assembled, and accumulated in their centres black holes of a billion solar masses.

How can we probe back observationally beyond redshifts 6 towards the predicted 'first light' at redshifts 20 or more, and thereby perhaps gain independent clues to the early growth of galaxies? There are various prospects in the next decade.

We do not expect any stellar systems at ultra-high redshifts to be as large and luminous as present-day galaxies. But there is hope of detecting these 'subgalaxies' if they are gravitationally lensed by nature's telescopes -clusters of galaxies along the line of sight.

Individual supernovae may outshine these subgalaxies, but their detection at redshift 10 may need to await JWST, or the next generation of ground-based telescopes. There is however one kind of object, much rarer than supernovae, that is certainly luminous enough to be found by large-area searches: gamma ray bursts. The Swift satellite has already detected two burst with $z$ above 6 : it could in principle record a burst at redshifts more than 10. Such ultra-remote objects would signify that massive stars indeed formed early -the gamma-ray bursts might signal the formation of the 'seed' black holes. 
If we cannot find discrete objects at these ultra-high redshifts, the best hope of probing the first 'action' when stars start to form may be to detect $21 \mathrm{~cm}$ emission from neutral hydrogen: it is a very weak signal compared to other radio backgrounds -around $0.01 \mathrm{~K}-$ but because Hi emits a line not a continuum, one can do 3-D mapping ('tomography') thereby probing the fraction of gas that remans neutral, and how it is clustered and ionized.

What happened before any structures formed? Even the more cautious among us are confident that our universe is the expanding aftermath of a "big bang" nearly 14 billion years ago. The most compelling evidence is that all space is warmed to 3 degrees above absolute zero by weak microwaves -the diluted and cooled afterglow of the hot dense beginning. That was over 40 years ago. Since that time the observations have huge improved. We now have a 'standard' model, where the early universe is characterised by a few numbers - the baryon/photon ratio, the density ratio of dark matter and baryons, and so forth.

And the theory has lived dangerously. It couldn't have been confidently foreseen that a consistent model would emerge. Here, for instance, are five things that could have happened, which would have invalidated any 'hot big bang' model:

(a) Discovery of an object with well below 23 percent helium;

(b) A millimetre-wave background with intensity below a black body curve fitted to the longer-wavelength background;

(c) A stable neutrino with mass in the range $100-10^{6}$ ev;

(d) Too much deuterium to match the CMB prediction for even the minimum observed baryon density;

(e) Angular fluctuations in the CMB temperature with amplitude too small to account for present-day clustering.

A special word about the last point: the best data on angular fluctuations come from the Wilkinson Microwave Anisotropy Probe (WMAP) which has for seven years been transmitting high-precision data from the Lagrangian point beyond the Moon. Computer simulations of an expanding universe taking these fluctuations (which are at a redshift of 1000) as input, lead to a present day universe which is a good quantitative match to actual observations on all scales from dwarf galaxies right up to superclusters. The amplitude of the fluctuations in the CMB characterises the amount of 'roughness' in the metric of early universe and determines the scale of present-day clustering. It is measured by a dimensionless number $Q$, whose value is about $10^{-5}(1 / 100,000)$.

\section{Cosmology and speculations}

We can divide cosmic history into three parts. The prevailing physics in the first trillionth of a second lies beyond the powers even of the LHC to simulate... This is the intellectual habitat of string theorists and inflationary cosmologists. But the phases from a millisecond to recombination (during which the light elements and the radiation spectrum formed) deserves to be taken as seriously as anything geophysicists tell us about the early history of our Earth. However, at some era after recombination, structures condense out, and evolve into the intricate asssemblage of galaxies, stars and planets we see around us. These 'recent' phases of cosmic evolution resemble other environmental sciences - the basic laws are known, but there's complicated nonlinearity and feedbacklike the weather.

We'd like to understand why the universe was 'set up', with its particular geometry, fluctuations, and mix of ingredients. The answer probably lies right back at the beginning - maybe even the first $10^{-44}$ second. Lawrence Krauss will be saying more about this 
later. But I'd like to venture a few comments -thought they should be preceded by a 'health warning' as they are speculative.

First, we might ask: How big is the universe? Is it even bigger than we can observe? We can only see a finite volume - a finite number of galaxies. That is essentially because there's a horizon, a 'shell' around us, delineating the distance light can have travelled since the big bang. But that shell has no more physical significance than the circle that delineates your horizion if you're in the middle of the ocean. We'd expect far more galaxies beyond the horizon.

Our universe seems destined to expand for ever, rather than to close up on itself within a few tens of billions of years. The fact that there is no perceptible gradient across the $20+$ billion light year domain that we observe suggests (by the ocean analogy) that we are nowhere near any 'edge'. The volume of space-time within range of our telescopes -what astronomers have traditionally called 'the universe'- could be only a tiny fraction of the aftermath of our big bang. Space very probably goes on much further. Indeed, space could go on for ever - more like an infinite 'flat earth' than a finite round one. Our universe could even, in principle, be vast enough to harbour an infinite number of duplicates of ourselves, taking all choices. In this universe, there would be room enough to exhaust all possibilities -absolutely everything that could happen would happen somewhere. Whenever we make a choice, a replica of ourselves (up till that point indistinguishable from us) takes the other branch.

But that's not all. "Our" big bang may not be the only one: one option is brane worlds -many universes embedded in a higher dimensional space (the 'bulk'). Bugs crawling on a large sheet of paper -their two dimensional universe- would be unaware of other bugs on a separate sheet of paper. Likewise, we would be unaware of our counterparts in another universe only a millimetre away. If that millimetre were measured in a 4th spatial dimension, and we are imprisoned in just three. Another is 'eternal inflation'. What we've traditionally called 'the universe' could be just one patch of space-time in a vast cosmic archipelago. This hugely expanded cosmic perspective takes Copernican modesty one stage further. To put this on a firm footing, we'll need a unified theory of all the forces.

The laws and constants of physics seem the same everywhere we can observe - even in the most distant galaxy. But the observable domain - what we've traditionally called 'the universe'- may be just an infinitesimal part of reality.

400 years ago, Kepler thought that the Earth was unique, and its orbit was a circle, related to the other planets by beautiful mathematical ratios. We now realise that even within our Galaxy there are probably billions of stars with planetary systems. Earth's orbit is special only insofar as it's in the range of radii and eccentricities compatible with life.

Maybe we are due for an analogous conceptual shift, on a far grander scale. Our big bang may not be unique, any more than planetary systems are. Its parameters may be 'environmental accidents', like the details of the Earth's orbit. In this enlarged cosmic perspective, what we have traditionally called fundamental constants and laws could be mere parochial bylaws in our cosmic patch. They might derive from some overarching theory governing the ensemble, but not be uniquely fixed by that theory. The hope for neat explanations in cosmology may be as vain as Kepler's numerological quest. Our universe isn't the neatest and simplest. It has the rather arbitrary seeming mix of ingredients -in the parameter range that allows us to exist.

We don't know if these theories are right. But they're speculative science, not metaphysics. What could give us confidence in unobservable domains? The answer's clear -we'll believe in them if they're predicted by a theory that gains credibility because it 
accounts for things we can observe? We believe in quarks, and in what general relativity says about the inside of black holes, because our inferences are based on theories corroborated in other ways. A challenge for 21st century physics is to decide whether there been many 'big bangs' rather than just one -and (if there are many) how much variety they might display.

\section{Summary comments}

Observational progress settles old controversies and enlarges consensus. But these advances bring into focus new questions that previously couldn't even have been posed. This talk has addressed some speculative areas: Earth-like planets, life, the flaky boundaries of cosmology. I chose to emphasise them at this conference because they are topics where the gap between generalist and specialist is smallest -we're all flummoxed. In the coming decades, there are three types of challenge.

The first is to seek to understand in some deep sense why our universe is the way it is. This involves 'fundamental' questions that may demand major conceptual advances in physics. But there is a second aim, which may not require new physics, but is as dauntingly complex as any environmental science: How, from simple beginnings described by a few parameters, did our universe transform itself into the cosmic habitat we see around us? We can identify the key stages in the emergence of structural complexity: the first particles (protons and neutrons); the first stars and galaxies; the synthesis of the periodic table in stars; and the formation of planets around later-generation stars. This opened the way, here on Earth, to the formation of a biosphere, and to the emergence of brains capable of pondering their origins. And there is a third question, most fascinating of all to most of us (as it was to Arthur C. Clarke): has this happened repeatedly, or is intelligent life unique to the Earth? The former outcome makes the universe more interesting, though the latter would boost our cosmic self-esteem. Either way, it is fascinating to ponder how much of what is now science fiction will become science fact. 